\title{
A high-throughput SNP discovery strategy for RNA-seq data
}

\author{
Yun Zhao, Ke Wang, Wen-li Wang, Ting-ting Yin, Wei-qi Dong and Chang-jie Xu* (D)
}

\begin{abstract}
Background: Single nucleotide polymorphisms (SNP) have been applied as important molecular markers in genetics and breeding studies. The rapid advance of next generation sequencing (NGS) provides a high-throughput means of SNP discovery. However, SNP development is limited by the availability of reliable SNP discovery methods. Especially, the optimum assembler and SNP caller for accurate SNP prediction from next generation sequencing data are not known.

Results: Herein we performed SNP prediction based on RNA-seq data of peach and mandarin peel tissue under a comprehensive comparison of two paired-end read lengths (125 bp and $150 \mathrm{bp}$ ), five assemblers (Trinity, IDBA, oases, SOAPdenovo, Trans-abyss) and two SNP callers (GATK and GBS). The predicted SNPs were compared with the authentic SNPs identified via PCR amplification followed by gene cloning and sequencing procedures. A total of 40 and 240 authentic SNPs were presented in five anthocyanin biosynthesis related genes in peach and in nine carotenogenic genes in mandarin. Putative SNPs predicted from the same RNA-seq data with different strategies led to quite divergent results. The rate of false positive SNPs was significantly lower when the paired-end read length was $150 \mathrm{bp}$ compared with $125 \mathrm{bp}$. Trinity was superior to the other four assemblers and GATK was substantially superior to GBS due to a low rate of missing authentic SNPs. The combination of assembler Trinity, SNP caller GATK, and the paired-end read length $150 \mathrm{bp}$ had the best performance in SNP discovery with 100\% accuracy both in peach and in mandarin cases. This strategy was applied to the characterization of SNPs in peach and mandarin transcriptomes.

Conclusions: Through comparison of authentic SNPs obtained by PCR cloning strategy and putative SNPS predicted from different combinations of five assemblers, two SNP callers, and two paired-end read lengths, we provided a reliable and efficient strategy, Trinity-GATK with $150 \mathrm{bp}$ paired-end read length, for SNP discovery from RNA-seq data. This strategy discovered SNP at 100\% accuracy in peach and mandarin cases and might be applicable to a wide range of plants and other organisms.
\end{abstract}

Keywords: Single nucleotide polymorphism (SNP), RNA-seq, Paired-end read length, Trinity, GATK

\section{Background}

Single nucleotide polymorphisms (SNPs) are single nucleotide base variations, caused by transitions $(\mathrm{C} / \mathrm{T}$ or $\mathrm{G} / \mathrm{A})$ or transversions $(\mathrm{C} / \mathrm{G}, \mathrm{C} / \mathrm{A}$, or $\mathrm{T} / \mathrm{A}, \mathrm{T} / \mathrm{G})$, in the same position between individual genomic DNA sequences [1, 2]. SNP is the predominant type of DNA polymorphism for genetic variation, which is ubiquitously located in genomes $[3,4]$ in the intergenic region (regions between genes), coding sequences of genes (exons), or non-coding regions of genes (introns, 5'UTR,

\footnotetext{
* Correspondence: chjxu@zju.edu.cn

Zhejiang Provincial Key Laboratory of Horticultural Plant Integrative Biology, Zhejiang University, Zijingang Campus, Hangzhou, China
}

3'UTR, or exon-intron splicing sites) [5]. SNPs in the coding region can be divided into two types, synonymous and nonsynonymous SNPs, with protein sequence affected by the latter type.

Considerable effects on protein function and gene expression can be caused by SNPs occurring in coding regions and regulatory sequences, respectively. Therefore, SNPs are of great potential in genetics, breeding, ecological and evolutionary studies [6]. Due to the high density, scalability and genome-wide distribution, SNPs are considered as ideal genomic resources in genetic studies for the characterization of genetic resources and functional gene identification for traits [7]. In plant

(c) The Author(s). 2019 Open Access This article is distributed under the terms of the Creative Commons Attribution 4.0 International License (http://creativecommons.org/licenses/by/4.0/), which permits unrestricted use, distribution, and 
genetics for instance, SNPs have been widely used to identify cis-regulatory variation within a species based on allele-specific assays and to discover genes linked to complex genetic traits.

With the rapid advances in modern sequencing technology, or next generation sequencing (NGS), de novo and reference-based SNP discovery is performed in numerous organisms, including many plants, even those where there is little or no genetic information available [8]. The availability of NGS provides a convenient approach to discover all SNPs and obtain relevant information on genomic position and genotyping in a single step. Data from reliable large-scale sequencing, especially in diploid plants [9], could improve the cost-effectiveness and efficiency of detection of abundant SNPs. A number of methods have been used for initial SNPs discovery in a high-throughput manner, such as whole-genome sequencing, exome capture, RNA sequencing, methylated DNA sequencing, and restriction enzyme (RE) digestion [10].

Recently, transcriptome sequencing, or RNA-seq, has become one of the most representative high-throughput sequence-based techniques because of its high accuracy and cost-effective. There are multiple advantages in carrying out SNP analysis using RNA-seq data. Firstly, thousands of SNPs can be discovered and the expression levels of functional genes with sequence variations can be observed simultaneously at a reasonable cost. Secondly, the location of variations in coding regions associated with plant biological and agronomical traits can be identified and the phenotypes can be predicted according to genotypes [11]. In addition, it is also a useful platform for related studies like gene characterization, gene expression quantification as well as post translational process analysis [12]. For these reasons, RNA-seq is developing into an extensive application in genetic polymorphisms analysis.

The basic procedures for converting raw data generated from whole genome or transcriptome sequencing into a final SNP result include obtaining raw data from NGS platforms, assembling, and SNP calling to identify SNP between the same unigenes or between different samples from different plant varieties or within one sample when the plant is genetically heterogeneous. Different NGS platforms such as Illumina Genome Analyzer, Roche/454 FLX and ABI SOLiD vary in terms of sensitivity, accuracy, reproducibility and throughput and this means that sequencing data obtained from different platforms has various advantages and limitations [13, 14]. At present, Illumina with good sequencing coverage and read quality, is applied to many plant species for SNP detection by RNA-seq. Currently, assemblers such as Trinity, IDBA, oases, SOAPdenovo and trans-abyss $[15,16]$ and SNP callers, or variant callers, Genome Analysis Toolkit (GATK) [17], Genotyping-by-sequencing (GBS) [18], SAMtools/
BCFtools [19], freebayes [20] and SOAPsnp [21], especially the former two callers, are the most extensively used. RNA-seq can produce massive SNPs at relatively low costs. On the other hand, omissions and errors are major obstacles for SNP identification from RNA-seq data. The availability of multiple choices for different read lengths, assemblers and SNP callers make the SNP analysis even more complicated. It was reported that different read lengths, assemblers and SNP callers all contribute to the accuracy and reliability of the final SNP result [22-25]. Development of existing technologies, especially increased read length, improves the quality of raw sequencing data and significantly reduces missing or erroneous SNPs caused by sequencing and assembling errors [25-27]. Therefore, theoretically a longer read length can produce higher quality raw data and influence downstream analyses [28-31], although no reports about the effects of read length on the accuracy of SNP discovery are available. Selection of a suitable assembler is also critical for SNP detection. For example, Jung's study on freshwater prawn showed that the results were seriously affected by the assemblers adopted, and an approach to obtain a comprehensive and reliable assembly was necessary [32]. The final SNP results were also affected by the SNP callers applied. For example, SNPs called individually from sequencing data from an Arabidopsis sup1ros1 ecotype by different tools (GeMS, SAMtools and GATK) led to distinct SNP accuracy rate in You's study [27]. A study about whole-genome sequencing of dairy cattle also showed that various SNP callers cause significant differences in the number of variants [33]. The SNP array at the whole-genome sequence level in chicken showed that appropriate SNP callers had high values in measures of SNPs especially for those with low allele frequency [24].

At present, the best assembling and SNP calling software combination for achieving the most accurate SNP data on RNA-seq is not known. The access to SNP information on RNA-seq data is a formidable task limited by the availability of reliable SNP discovery methods including assembling and SNP calling pipeline to resolve the problems of genotyping errors and missing data. It is therefore crucial to establish a reliable strategy to overcome the limitations in RNA-seq for downstream SNP analyses.

Woody fruit crops are propagated asexually and therefore have a genetic background of high heterozygosity and maintain a genetic resource of high diversity. In this study, two peach cultivars ('Hujingmilu' with deep red appearance and 'Yulu' with barely pigmented appearance [34]) and two mandarin cultivars ('Ponkan' with orange-reddish appearance and 'Yellowish-peeled Ponkan' with yellowish appearance [35]) were used for SNP detection. Since anthocyanins and carotenoids are characteristic pigments for ripe peach and mandarin, a study 
of the presence of SNPs in pigment biosynthesis genes can contribute to the understanding of the possible mechanisms for differential coloration in ripe fruit peel.

Here transcriptome sequencing of two peach cultivars and two mandarin cultivars was completed and for peach, two paired-end read lengths was involved. The raw data were processed with five common assemblers and two SNP callers and predicted SNPs were generated. The predicted SNPs inside a number of selected genes of peach (genes involved in anthocyanin biosynthesis) and mandarin (genes involved in carotenoid biosynthesis) were compared with authentic ones identified via PCR amplification, gene cloning and sequencing procedures. The effects of different paired-end read lengths, assemblers, and SNP callers on the accuracy of SNP results were investigated and it was found that SNPs can be accurately discovered by performing RNA-seq with a $150 \mathrm{bp}$ read length, assembling with Trinity and SNP calling with GATK. The study provides general guidelines for accurate SNP discovery from transcriptome data.

\section{Results}

\section{Overview of transcriptome sequencing}

Transcriptome sequencing of peach cultivars 'Hujingmilu' ('HJ') and 'Yulu' ('YL'), and mandarin cultivars 'Ponkan' ('PK') and 'Yellowish-peeled Ponkan' ('YP') was performed by Illumina HiSeq 2500 and 4000 (the raw data were deposited in NCBI under accession number SRP155137). Paired-end read lengths of $125 \mathrm{bp}$ and 150 bp were applied to peach and $150 \mathrm{bp}$ to mandarin. A summary of the sequencing statistics is shown in Table 1. A total of $40.7 \mathrm{G}$ data, on average $6.7 \mathrm{G}$ data for each library, were obtained. A collection of over 20 million high-quality clean reads were obtained from each library with the Q20 > 92\% (Table 1). Quality Score showed the corresponding incorrect base calling in each library was less than $0.01 \%$. The GC dinucleotide content was over $44 \%$. After pre-processing filtering of low-quality sequences and adaptor trimming, high quality sequencing reads that passed thresholds were assembled for further SNP discovery analysis.

\section{De novo assembly and transcriptome annotation}

Reads filtered, processed and assembled into contigs as described above were used for SNP discovery. To find an optimum assembly method for SNP detection purposes, the six libraries were analysed using five different de novo assemblers including Trinity, IDBA, oases, SOAPdenovo, Trans-abyss. Due to the different standards of contig length and N50 length applied by the five assemblers, different assembly outcomes were generated. The length of unigenes ranged from $200 \mathrm{bp}$ to $3000 \mathrm{bp}$ for all five assemblers. Taking Trinity as an example, clean reads from three libraries were assembled de novo into 106,574, 103,342, 167,580 transcripts with a mean size of $1545 \mathrm{bp}$, and $44,470,49,235,66,148$ unigenes with a mean size of 856 bp, respectively (Additional file 1: Table S1). A summary of the detailed length distribution of transcripts and unigenes is listed in Additional file 1: Table S1.

The alignment results with six libraries using Trinity are shown in Additional file 2: Table S2. Over $76 \%$ of total clean reads were mapped to associated unigenes in each library (Additional file 2: Table S2), suggesting that the quality of unigene data was high enough for subsequent SNP calling procedures. When the paired-end read length was $125 \mathrm{bp}$, the percentages of multi-mapped reads were approximately $60 \%$ in both ' $\mathrm{HJ}$ ' and 'YL' and that of uniq-mapped reads were around $40 \%$ (Additional file 2: Table S2). The percentages of multi-mapped reads declined dramatically to less than $30 \%$, meanwhile, the percentages of uniq-mapped reads were significantly higher when the paired-end read length was $150 \mathrm{bp}$ (Additional file 2: Table S2). These data implied a strong influence of paired-end read length on read mapping quality.

\section{Single nucleotide polymorphism prediction}

As illustrated in Fig. 1, two SNP callers (GATK and GBS) were applied to analyse RNA-seq data for SNP discovery following assembly with five different assemblers. We processed SNP calling under parameters described in the Methods section to maximize reliable SNPs and minimize omissions and false-positive SNPs. After calling peach and mandarin transcriptome data with GATK and GBS, predicted SNPs were recorded. Depending on the paired-end read lengths, and the assemblers and

Table 1 Summary of the sequencing data of 'Hujingmilu' ('HJ') and 'Yulu' ('YL') peach and 'Ponkan' ('PK') and 'Yellowish-peeled Ponkan' ('YP') mandarin libraries with either 125 bp or $150 \mathrm{bp}$ paired-end read lengths

\begin{tabular}{lllllll}
\hline Samples & Clean Read Number & Base Number & GC Content (\%) & N (\%) & Q20 (\%) & Q30 (\%) \\
\hline HJ-125 bp & $22,182,670$ & $5,585,820,171$ & 45.96 & 0.01 & 92.64 & 87.38 \\
YL-125 bp & $20,122,397$ & $5,066,451,719$ & 46.06 & 0.01 & 92.69 & 97.41 \\
HJ-150 bp & $25,905,927$ & $7,736,134,266$ & 46.14 & 0.00 & 95.46 & 93.88 \\
YL-150 bp & $21,836,682$ & $6,522,644,616$ & 46.48 & 0.00 & 86.74 \\
PK-150 bp & $29,498,009$ & $8,784,662,004$ & 44.48 & 0.00 & 96.74 & 92.90 \\
YP-150 bp & $23,641,003$ & $7,038,555,478$ & 44.34 & 0.00 & 96.79 \\
\hline
\end{tabular}




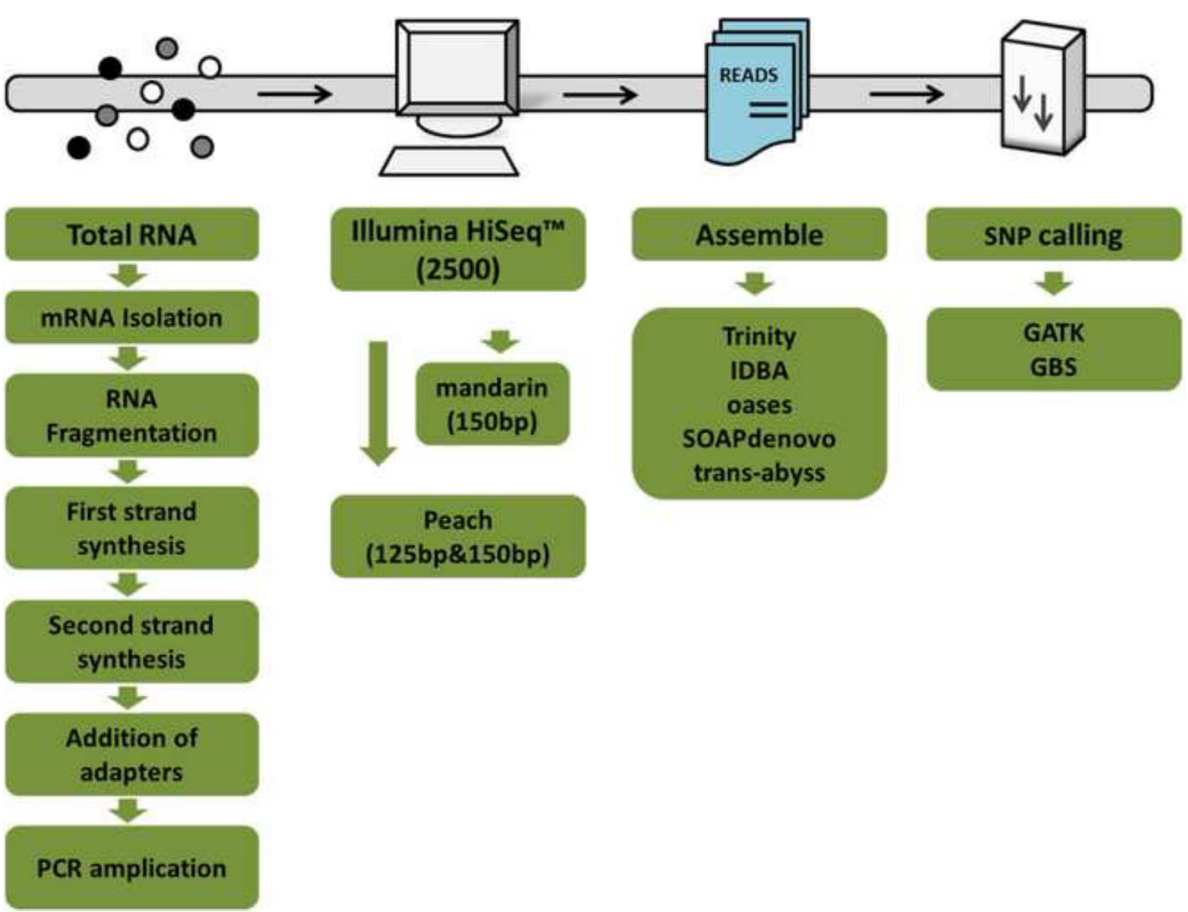

Fig. 1 A simplified workflow of analysis strategies for RNA-seq and SNP discovery. The clip arts are drawn with PowerPoint 2010

SNP callers employed, 7752-56,271 and 43,944-127,201 SNPs were found residing in peach and mandarin transcriptomes, respectively (Additional file 3: Table S3). Comparing data from two SNP calling methods, GATK showed a significantly (1.2-3.5 times) higher number of SNP than GBS in all libraries from five assemblers employed (Additional file 3: Table S3). The data from two paired-end read lengths (125 bp and $150 \mathrm{bp}$ ) in peach were compared and it was found that the number of SNP predicted with the same assembler and SNP caller was affected by read lengths. For four assemblers, except for oases, the number of SNPs predicted was higher when the read length was $125 \mathrm{bp}$ (Additional file 3: Table S3). Irrespective of the read lengths and SNP callers employed, the minimum number of SNPs was generated with assembler IDBA_tran for both peach and mandarin (Additional file 3: Table S3).

\section{Identification and validation of putative SNPs in selected genes}

To explore which assembler and SNP caller were most suitable for accurately detecting SNPs, the SNPs predicted through transcriptomic analysis were compared with those obtained via PCR amplification followed by gene cloning and sequencing procedures. Five anthocyanin biosynthesis related genes in peach and nine carotenogenic genes in mandarin possessing putative SNPs as predicted by at least one combination of read length, assembler and SNP caller were selected. The authentic
SNPs presented inside these genes were obtained following PCR, gene cloning and sequencing procedures (Additional file 4: Table S4 and Additional file 5: Table S5).

A total of 40 authentic SNPs were identified in the five anthocyanin biosynthesis related genes (CHS, DFR, ANS, UFGT and WD40-1) of peach (Additional file 4: Table S4), and the number of SNPs predicted through different combinations of assemblers and SNP callers for transcriptome analysis as well as the accuracy are summarized in Table 2. Five assemblers and two SNP callers were applied in this study, which made up ten combinations in total. All these combinations were applied to transcriptomic data from paired-end read lengths of 125 bp and $150 \mathrm{bp}$, respectively, and an overview of raw data for targeted genes listed in Additional file 6: Table S6 and Additional file 7: Table S7.

The accuracy of SNP discovery in peach was compared between ten combinations of assemblers and SNP callers. Different read lengths under the same assemblers and SNP callers resulted in distinct results for the same samples. The accuracy of SNP discovery, represented by the higher percentage of true SNP discovered and a much lower percentage of false positive SNP predicted, was higher with a read length of $150 \mathrm{bp}$ compared to 125 bp (Table 2). For ten combinations, the average percentage of true SNP discovery was 50.25 and $39.25 \%$ respectively when the read lengths was $150 \mathrm{bp}$ and $125 \mathrm{bp}$, while the percentage of false positive SNP discovery was 6.11 and $34.45 \%$ (Table 2). Moreover, with a read length 
Table 2 Accuracy of SNP predictions from ten combinations of assemblers and SNP callers with 40 authentic SNPs presented in five anthocyanin biosynthesis related genes in peach as example. The RNA-Seq was performed under the read lengths of 125 bp and $150 \mathrm{bp}$

\begin{tabular}{|c|c|c|c|c|c|c|c|c|}
\hline No. & $\begin{array}{l}\text { Paired-end read } \\
\text { length (bp) }\end{array}$ & Assembler & SNP caller & $\begin{array}{l}\text { Authentic SNPs } \\
\text { presented }\end{array}$ & $\begin{array}{l}\text { True SNPs } \\
\text { discovered }\end{array}$ & $\begin{array}{l}\text { True SNPs } \\
\text { rate (\%) }\end{array}$ & $\begin{array}{l}\text { False positive } \\
\text { SNPs predicted }\end{array}$ & $\begin{array}{l}\text { False positive } \\
\text { SNPs rate (\%) }\end{array}$ \\
\hline 1 & 125 & Trinity & GATK & 40 & 25 & 62.50 & 11 & 30.56 \\
\hline 2 & 125 & Trinity & GBS & 40 & 14 & 35.00 & 10 & 41.67 \\
\hline 3 & 125 & IDBA_tran & GATK & 40 & 28 & 70.00 & 16 & 36.36 \\
\hline 4 & 125 & IDBA_tran & GBS & 40 & 0 & 0 & 0 & 0 \\
\hline 5 & 125 & oases & GATK & 40 & 32 & 80.00 & 40 & 55.56 \\
\hline 6 & 125 & oases & GBS & 40 & 3 & 7.50 & 9 & 75.00 \\
\hline 7 & 125 & SOAPdenovo & GATK & 40 & 23 & 57.50 & 5 & 17.86 \\
\hline 8 & 125 & SOAPdenovo & GBS & 40 & 5 & 12.50 & 3 & 37.50 \\
\hline 9 & 125 & Trans-abyss & GATK & 40 & 24 & 60.00 & 8 & 25.00 \\
\hline 10 & 125 & Trans-abyss & GBS & 40 & 3 & 7.50 & 1 & 25.00 \\
\hline Average & 125 & / & / & 40 & 15.70 & 39.25 & 10.30 & 34.45 \\
\hline 11 & 150 & Trinity & GATK & 40 & 40 & 100.00 & 0 & 0 \\
\hline 12 & 150 & Trinity & GBS & 40 & 6 & 15.00 & 0 & 0 \\
\hline 13 & 150 & IDBA_tran & GATK & 40 & 28 & 70.00 & 4 & 12.50 \\
\hline 14 & 150 & IDBA_tran & GBS & 40 & 6 & 15.00 & 2 & 25.00 \\
\hline 15 & 150 & oases & GATK & 40 & 39 & 97.50 & 1 & 2.50 \\
\hline 16 & 150 & oases & GBS & 40 & 6 & 15.00 & 0 & 0 \\
\hline 17 & 150 & SOAPdenovo & GATK & 40 & 35 & 87.50 & 3 & 7.89 \\
\hline 18 & 150 & SOAPdenovo & GBS & 40 & 6 & 15.00 & 0 & 0 \\
\hline 19 & 150 & Trans-abyss & GATK & 40 & 33 & 82.50 & 5 & 13.16 \\
\hline 20 & 150 & Trans-abyss & GBS & 40 & 2 & 5.00 & 0 & 0 \\
\hline Average & 150 & / & / & 40 & 20.10 & 50.25 & 1.50 & 6.11 \\
\hline
\end{tabular}

of $125 \mathrm{bp}$, a combination with a higher accuracy was often accompanied by a higher error rate, e.g., Oases-GATK had the highest accuracy of $80.00 \%$, but its error rate was also highest, up to $55.56 \%$ (Table 2). There was no obvious correlation between the percentage of authentic SNP discovery and the percentage of false positive SNP discovery with read length $150 \mathrm{bp}$ (Table 2). The accuracy of SNP discovery was also affected by the combinations of assemblers and SNP callers. When the read length was $150 \mathrm{bp}$, the combination of Trinity and GATK produced the highest accuracy, i.e., 100\% discovery of authentic SNP and no prediction of any false positive SNP (Table 2). No combination with $100 \%$ accuracy was observed when the read length was $125 \mathrm{bp}$, further indicating the importance of longer read length. For read length $150 \mathrm{bp}$, no false positive SNP was predicted with four assemblers (with the single exception of IDBA) when the SNP caller GBS was taken. Compared with GATK, the high filtration standard of GBS reduced the error rate, but on the other side, missed a lot of authentic SNPs and the rate of true SNP discovery dropped to $15 \%$ with four assemblers and to $5 \%$ with the assembler Trans-abyss (Table 2). For GATK, the accuracy with five assemblers reached $70 \%$ or above. However, only the combination Trinity-GATK reached $100 \%$ accuracy and no false positive SNP prediction, while IDBA-GATK had the lowest accuracy of $70 \%$ and trans-abyss-GATK had the highest false positive SNP rate of $13.16 \%$ (Table 2 ). The study showed that not only SNP callers, but also assemblers had a strong influence on accuracy of SNP discovery from RNA-seq data.

To investigate whether the optimum combination of assembler and SNP caller is independent of plant species, all ten combinations were also applied to transcriptomic data from mandarin with read length of $150 \mathrm{bp}$. Nine carotenogenic genes (ZEP, PSY1, PSY2, BCH1, $B C H 3, V D E, L C Y B, C Y C B, C C D 1)$ were chosen to validate. A total of 240 authentic SNPs were identified, via PCR amplification and gene cloning and sequencing strategy (Additional file 5: Table S5), and the SNPs predicted, via bioinformatic strategy, are listed in Additional file 8: Table S8. A summary of the number of SNPs predicted through different combinations of assemblers and SNP callers for transcriptome analysis as well as the accuracy were summarized in Table 3. 
Table 3 Accuracy of SNP predictions from ten combinations of assemblers and SNP callers with 240 authentic SNPs presented in nine carotenogenic genes in mandarin as example. The RNA-Seq was performed under the read length of $150 \mathrm{bp}$

\begin{tabular}{|c|c|c|c|c|c|c|c|c|}
\hline No. & $\begin{array}{l}\text { Paired-end read } \\
\text { length (bp) }\end{array}$ & Assembler & SNP caller & $\begin{array}{l}\text { Authentic SNPs } \\
\text { presented }\end{array}$ & $\begin{array}{l}\text { True SNPs } \\
\text { discovered }\end{array}$ & True SNPs rate (\%) & $\begin{array}{l}\text { False positive } \\
\text { SNPs predicted }\end{array}$ & $\begin{array}{l}\text { False positive } \\
\text { SNPs rate }(\%\end{array}$ \\
\hline 1 & 150 & Trinity & GATK & 240 & 240 & 100.00 & 0 & 0 \\
\hline 2 & 150 & Trinity & GBS & 240 & 122 & 50.83 & 0 & 0 \\
\hline 3 & 150 & IDBA_tran & GATK & 240 & 32 & 13.33 & 2 & 5.88 \\
\hline 4 & 150 & IDBA_tran & GBS & 240 & 25 & 10.42 & 1 & 3.85 \\
\hline 5 & 150 & oases & GATK & 240 & 122 & 50.83 & 12 & 8.96 \\
\hline 6 & 150 & oases & GBS & 240 & 48 & 20.00 & 6 & 11.11 \\
\hline 7 & 150 & SOAPdenovo & GATK & 240 & 129 & 53.75 & 7 & 5.15 \\
\hline 8 & 150 & SOAPdenovo & GBS & 240 & 88 & 36.67 & 14 & 13.73 \\
\hline 9 & 150 & Trans-abyss & GATK & 240 & 193 & 80.42 & 13 & 6.31 \\
\hline 10 & 150 & Trans-abyss & GBS & 240 & 94 & 39.17 & 6 & 6.00 \\
\hline Average & 150 & / & I & 240 & 109.30 & 45.54 & 6.10 & 6.10 \\
\hline
\end{tabular}

Similar to the findings on peach, only the combination of Trinity with GATK produced $100 \%$ accuracy in the mandarin case, followed by trans-abyss-GATK with an accuracy rate of $80.42 \%$ (Table 3). Among all SNP discovery strategies, the GBS caller always filtered out a lot of true SNPs and the rate of true SNP discovered was substantially inferior to GATK. This is consistent with the findings from peach data analysis. In summary, the combination of Trinity with GATK was the best strategy for SNP discovery, obtaining 100\% accuracy in peach and mandarin when the read length was $150 \mathrm{bp}$, and this strategy might be applicable to wide range of plants and other organisms.

\section{Characterization of SNPs in peach and mandarin transcriptomes}

As described above, using the combination of Trinity with GATK and with a read length of $150 \mathrm{bp}$, SNPs in transcriptome data of peach and mandarin can be accurately discovered. Here, with such strategy, further characterization of SNPs was performed in the four transcriptomes from two peach cultivars and two mandarin cultivars. The numbers of SNPs predicted is shown in Fig. 2. The number of total SNPs varied only slightly, less than $1.2 \%$, between two cultivars, but was relatively greater between two species, with the number being 2.58 times higher in mandarin than in peach. Overall, the SNP density in mandarin was higher than in peach (Additional file 9: Figure S1).

SNPs can be sorted into two types, including homozygous types for those SNPs presented between cultivars but not between two alleles of an individual cultivar, and heterozygous types for those SNPs presented between two alleles of an individual cultivar. As shown in Fig. 2, the number of heterozygous SNPs is higher than homozygous ones in all four transcriptomes. For all SNPs found in peach cultivars ' $\mathrm{HJ}$ ' and ' $\mathrm{YL}$ ', respectively, 18,072 and 20,248 were heterozygous (HZ) while 15,166 and 12,617 were homozygous (HM), with an average HZ:HM proportion of 1.39:1 in peach. Over three times of SNPs were found in mandarin, compared with peach, and many more heterozygous SNPs (106,466 and 104,732) were found than homozygous SNPs $(12,213$ and 13,311) with an averaged HZ:HM proportion of 8.29:1 in mandarin (Fig. 2).

For the heterozygous SNPs in all four transcriptomes, fewer transversion substitutions (four possibilities, 38.33 to $40.05 \%$ ) were found than transitions substitution (two possibilities, 59.95 to $61.67 \%$ ) (Additional file 10: Figure S2). Transition substitutions significantly outnumbered the transversion substitutions by almost a factor of 2 (Additional file 10: Figure S2). Nearly even distribution between two variation types of transitions (G/A, C/T), and to a lesser extent, between four variation types of transversion (C/A, C/G, T/G, T/A), are observed for the 'HJ' transcriptome (Additional file 10: Figure S2). Similar results were found with the other three transcriptomes. Moreover, the distribution patterns of substitution types were also similar between peach and mandarin (Additional file 10: Figure S2), indicating presence of a conserved mechanism during evolution.

\section{Discussion}

RNA-seq dataset and following SNP discovery often differ due to sequencing quality as affected by the read length, sequencing depth and sequencing platforms as well as various downstream analyses [13, 14, 28]. In this study, our data suggested that the accuracy of SNP discovery was affected by paired-end read lengths, assemblers and SNP callers. Previously the effect of read length on accuracy of SNP discovery has not reported, although it was suggested that a higher read length can 


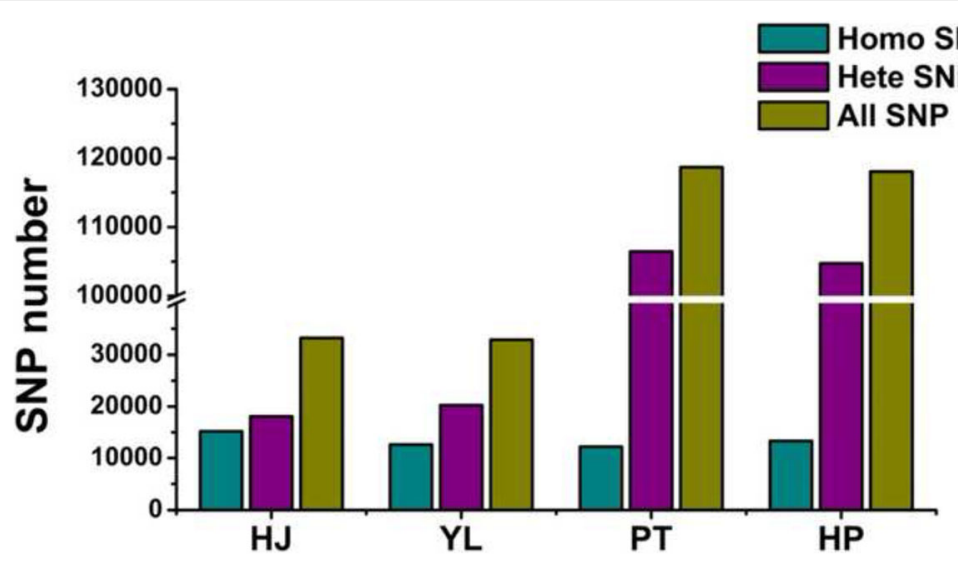

Fig. 2 The numbers of heterozygous (purple) and homozygous (cyan) SNPs discovered in peach (cv. HJ and cV. YL) and mandarin (cv. PK and cV. YP) transcriptomes using Trinity and GATK with read length of $150 \mathrm{bp}$

improve the quality of NGS raw data [31]. Here our data indicated that the longer read length is necessary for high quality SNP discovery. More SNPs were found with 125 bp sequencing, but with higher rate of incorrect prediction compared with $150 \mathrm{bp}$ sequencing, resulting in greatly reduced accuracy with the shorter reads. The reasons for more false positive SNPs predicted from $125 \mathrm{bp}$ sequencing can be attributed mainly to a slightly higher sequencing error and an obviously higher rate of incorrect read mapping. As shown in Table 1, the sequencing error rate (N\%) for $\mathrm{HJ}-125 \mathrm{bp}, \mathrm{YL}-125 \mathrm{bp}$ was $0.01 \%$ while those for HJ-150 bp, YL-150 bp and PK-150 bp YP-150 bp were $0.00 \%$. Additional file 2: Table S2 showed a lower rate of uniq-mapped Reads and a higher rate of multi-mapped Reads for $125 \mathrm{bp}$ sequencing. Mapping to multiple positions can cause erroneous SNPs. Differences between results from different assemblers were observed as well and these can be attributed to the different methods for dealing with sequencing errors, resolving inconsistencies and using paired-end information [32]. Different assemblers vary in the details of usability (ability of installation and execution, speed) and assembly quality (the contiguity and the accuracy), which determine how they resolve errors and inconsistencies in the dataset, leading to distinct assembly performance [14]. Therefore, a suitable assembler is critical in order to improve the quality and performance of de novo assembly. The third factor affecting SNP discovery we found in this study is SNP caller. It was found that each SNP caller has its own characteristics and limitations. For example, although GBS, a stringent SNP caller, can efficiently avoid false SNPs, it easily results in omission because of its limitations such as genotyping errors, missing data and the under-calling of heterozygous sites. The study here revealed the merits and defects of two paired-end read lengths, five assemblers and two SNP callers for SNP analysis, and provide detailed comparison of different methods for reference.
Peach and mandarin are fruit crops propagated asexually and therefore the presence of a high number of heterozygous SNPs (Fig. 2) is not unexpected. The genetic heterogeneity of an organism can be represented by the rate of heterozygous SNP in the genome, and can also, to a large extent, be revealed in the transcriptome as well. The rate of heterozygous SNP among transcriptomes, calculated by dividing the number of heterozygous SNPs with the total length of unigenes (presented in Additional file 2: Table S2), was 0.045 and $0.202 \%$ respectively for peach and mandarin. The high rate of heterozygous SNPs in 'PK' and 'YP' mandarins suggested that these two mandarins are not pure mandarins. This is consistent with the recent findings that ' $\mathrm{PK}$ ' is an early-admixture mandarin containing a small amount of pummelo admixture [36]. Besides, it was found more heterozygous SNPs presented as transition substitutions than transversion substitutions (Additional file 10: Figure S2). This is in consistent with the conclusions that transition mutations, caused by the hypermutability effects of $\mathrm{CpG}$ dinucleotide sites and deamination of methylated cytosines, are the most frequent class of SNPs in both plant and animal genomes [37].

\section{Conclusions}

SNP is extensively used as a molecular marker for analysing genotype and trait association as well as assisting breeding. Traditional SNP mining techniques are generally low throughput and technically complicated. High efficient SNP discovery become possible in recent decades due to the availability of huge raw data from next generation sequencing. Although several assemblers and SNP callers have been used in previous literatures, the accuracy was rarely evaluated and the optimum combination of assembler and SNP caller is not clear. In addition, the effect of paired-end read length of sequencing on the accuracy of SNP discovery has not been 
reported. Here we evaluated the accuracy of SNP discovery from combinations of two paired-end read lengths, five assemblers, and two SNP callers, and established an ideal strategy, i.e., obtaining sequencing raw data from $150 \mathrm{bp}$ paired-end read length, assembling with Trinity and SNP calling with GATK, for SNP discovery. This strategy was proven to be $100 \%$ accurate for a total of 280 authentic SNPs analysed, while none of the other strategies reached $100 \%$ accuracy. The evaluation was carried out with transcriptome data from peel tissues of two peach cultivars and two mandarin cultivars, but the strategy should also be applicable to other organisms and tissues. With the advantages of high throughput, high accuracy, low cost, and especially, being independent of a reference genome, the method established here can be expected to be widely used for SNP discovery in genetic diversity analysis, breeding and genome-wide association studies.

\section{Methods}

\section{Plant materials}

Ripe fruit and young leaves of 'Hujingmilu' ('HJ') and 'Yulu' ('YL') peach [Prunus persica (L.) Batsch] as well as 'Ponkan' ('PK') and 'Yellowish-peeled Ponkan' ('YP') mandarin (Citrus reticulata Blanco) were collected at the Fenghua Peach Research Institute and Quzhou Fruit Science Institute, Zhejiang, China, respectively. All these materials are commercial cultivars with voucher specimens available in the above-mentioned institutes. Peach cultivars 'YL' and ' $\mathrm{HJ}$ ' were identified by Yin-chong Zhang in 1883 and A-pan Shao in 1964, respectively [38]. 'PK' mandarin is a natural cultivar with a history of over a hundred years and first identifier unknown. 'YP' mandarin was identified by Quzhou Bureau of Agriculture Economic Specialty Station in 2008 [35]. The peel tissues, after separation from the fruit, and the young leaves were frozen in liquid nitrogen on the day of collection and stored at $-80^{\circ} \mathrm{C}$ for further DNA or RNA extraction.

\section{DNA and RNA extraction}

Young leaves were ground to a fine powder in liquid nitrogen and the genomic DNA was isolated using an improved CTAB method [39]. Peel tissues were also ground to a fine powder in liquid nitrogen and the total RNA was isolated by a CTAB protocol as described by Shan [40] and treated with DNA-free DNA Removal kit (Invitrogen, Life Technologies, CA, USA) to remove DNA contamination. The concentrations of RNA samples were assessed by Qubit (Life Technologies, CA, USA). RNA purity was estimated with the NanoPhotometer ${ }^{\circ}$ spectrophotometer (IMPLEN, CA, USA) and integrity was evaluated by the RNA Nano 6000 Assay Kit of the Agilent Bioanalyzer 2100 system (Agilent Technologies, CA, USA).

\section{Library construction and transcriptome sequencing}

The library construction and transcriptome sequencing was carried out by staff at Biomarker (Beijing, China). In brief, the library was constructed following mRNA enrichment, mRNA fragmentation, second strand cDNA synthesis, adaptor ligation, size selection, PCR amplification and transcriptome sequencing. The sequencing was performed on an Illumina $\mathrm{HiSeq}^{\text {ti }} 2500$ and 4000 platform and paired-end reads were generated. Paired-end read lengths of $125 \mathrm{bp}$ and $150 \mathrm{bp}$ were used for peach cultivars and the length $150 \mathrm{bp}$ for mandarin cultivars. All raw data (raw reads) obtained were further processed through filtering parameters. After removing reads containing adapter or ploy-N and low-quality reads (Q-value $\leq 10)$ from raw data, high-quality clean data (clean reads) collected in FASTQ format were used for subsequent analyses. Clean data was further analysed to obtain Q20, Q30, GC content and sequence duplication level for quality control.

\section{Transcriptome assembly and SNP detection}

Five prevalent assemblers, i.e., Trinity, IDBA, oases, SOAPdenovo, Trans-abyss, were applied in all six transcriptome for de novo assembly. All of the generated unigenes were subjected to match against (E-value $\leq 1.0 \mathrm{e}-5$ ) public databases (Nr, Swiss-Prot, KEGG, COG, GO) and annotation information was retrieved. The clean reads exported in FASTQ format were aligned to unigenes through STAR software. Subsequently two widely used SNP callers, i.e., GATK and GBS, were applied to perform prediction of SNP site. Reads were processed with a further filtering step under GATK and GBS filter parameters (read depth no less than 10, quality score no less than 20, consecutive single base errors no more than 3 in $35 \mathrm{bp}$, FS 20.0 window 25). Only SNPs after sequential depth standardization with quality value $>2$ were retained. With all programs mentioned, the study resulted in ten different variant calling strategies: Trinity_GATK, IDBA_GATK, oases_GATK, SOAPdenovo_GATK, Trans-abyss_GATK and Trinity_GBS, IDBA_GBS, oases_GBS, SOAPdenovo_GBS, Trans-abyss_GBS. All SNPs were analysed within cultivars and then compared between cultivars. A simplified workflow of assembly and SNP calling is outlined in Fig. 1.

\section{Identification of SNPs through gene cloning procedures}

Genes related to anthocyanin biosynthesis in peach and carotenoid biosynthesis in mandarin were evaluated and the genes containing putative SNP, predicted by at least one variant calling strategy, were chosen for SNP identification through PCR amplification and gene cloning procedures [41]. Briefly, with Faststart High Fidelity PCR system (Roche, SUI), each PCR reaction mixture $(25 \mu \mathrm{L}$ total volume) contained $20 \mathrm{ng}$ genomic DNA, $0.2 \mu \mathrm{M}$ of 
each PCR primer $(10 \mu \mathrm{M}$, Additional file 11: Table S9), $0.1 \mathrm{mM}$ dNTPs, PCR buffer and 0.25 unit of DNA polymerase. PCR was performed with the following cycling conditions: $95^{\circ} \mathrm{C}$ for $5 \mathrm{~min}$ and 35 cycles of $95^{\circ} \mathrm{C}$ for $30 \mathrm{~s}, 58^{\circ} \mathrm{C}$ for $30 \mathrm{~s}$, and $72{ }^{\circ} \mathrm{C}$ for $60 \mathrm{~s} / \mathrm{kb}$, followed by a final elongation step of $72^{\circ} \mathrm{C}$ for $10 \mathrm{~min}$. The PCR products were cloned into pGEM-T Easy (Promega, USA), and for each gene, at least 15 recombinant plasmids were sequenced and sequence alignment was carried out with Seqman.

\section{Additional files}

Additional file 1: Table S1. Assembly statistics of peach and mandarin transcriptomes under Trinity. (DOCX $17 \mathrm{~kb}$ )

Additional file 2: Table S2. Summary of read mapping of peach ( $\mathrm{CV}$. HJ and $\mathrm{CV}$. YL) and mandarin ( $\mathrm{c}$. PK and $\mathrm{c}$. Y YP) transcriptomes under Trinity. (DOCX $18 \mathrm{~kb})$

Additional file 3: Table S3. Number of SNPs predicted from RNA-seq data under different paired-end read lengths, assemblers and SNP callers. The number is the average of two cultivars. (DOCX $17 \mathrm{~kb}$ )

Additional file 4: Table S4. The detailed information of 40 authentic SNPs in five anthocyanin biosynthesis related genes in peach. (DOCX $17 \mathrm{~kb}$ )

Additional file 5: Table S5. The detailed information of 240 authentic SNPs in nine carotenogenic genes in mandarin. (DOCX $21 \mathrm{~kb}$ )

Additional file 6: Table S6. An overview of the number of SNPs predicted in targeted genes from peach with ten different strategies under the read length of $125 \mathrm{bp}$. Values in brackets denote the ratio of heterozygous and homozygous SNP (HZ:HM). (DOCX $19 \mathrm{~kb}$ )

Additional file 7: Table S7. An overview of the number of SNPS predicted in targeted genes from peach with ten different strategies under the read length of $150 \mathrm{bp}$. Values in brackets denote the ratio of heterozygous and homozygous SNP (HZ:HM). (DOCX $19 \mathrm{~kb}$ )

Additional file 8: Table S8. An overview of the number of SNPs predicted in targeted genes from mandarin with ten different strategies under the read length of $150 \mathrm{bp}$. Values in brackets denote the ratio of heterozygous and homozygous SNP (HZ:HM). (DOCX $22 \mathrm{~kb}$ )

Additional file 9: Figure S1. SNP density in unigene of peach ( $\mathrm{CV}$. HJ and $\mathrm{cV}$. YL) (A) and mandarin (cv. PK and cv. YP) (B) libraries using Trinity and GATK with read length of $150 \mathrm{bp}$. (JPG $1491 \mathrm{~kb}$ )

Additional file 10: Figure S2. Classification of heterozygous SNPS predicted in peach (cV. HJ and $\mathrm{cv}$. YL) and mandarin (cv. PK and cV. YP) libraries using Trinity and GATK with read length of $150 \mathrm{bp}$. (JPG $1763 \mathrm{~kb}$ )

Additional file 11: Table S9. Primers used for PCR amplification of five anthocyanin biosynthesis related genes in peach and nine carotenogenic genes in mandarin. (DOCX $17 \mathrm{~kb}$ )

\section{Abbreviations}

ANS: Anthocyanidin synthase; BCH1: Beta-carotene 3-hydroxylase 1, BCH3: Beta-carotene 3-hydroxylase 3; CCD1: Carotenoid cleavage dioxygenase 1; CHS: Chalcone synthase; COG: The clusters of orthologous groups of proteins database; CYCB: Capsorubin synthase:

DFR: Dihydroflavonol 4-reductase; GATK: Genome Analysis Toolkit; GBS: Genotyping-by-sequencing; GO: Gene Ontology enrichment database; HJ: Hujingmilu; KEGG: The Kyoto encyclopedia of genes and genomes database; LCYB: Lycopeneß-cyclase; NCBI: National center for biotechnology information; NGS: Next-generation sequencing: Nr: NCBI non-redundant database; PK: Ponkan; PSY1: Phytoene synthase 1; PSY2: Phytoene synthase 2; RNA-Seq: RNA-sequencing; SNP: Single nucleotide polymorphisms; SwissProt: Swiss-Prot protein database; UFGT: UDP-glucose: flavonoid 3-Oglucosyltransfersae; VDE: Violaxanthin de-epoxidase; YL: Yulu; YP: Yellowishpeeled Ponkan; ZEP: Zeaxanthin epoxidase

\section{Acknowledgements}

We would like to thank Prof. Don Grierson from the University of Nottingham (UK) for his efforts in language editing.

\section{Funding}

This study was financially supported by the National Natural Science Foundation in China (31572102), the Zhejiang Provincial Science and Technology Project for the Belt and Road (2016C04001) and the 111 project (B17039). The funding bodies did not contribute in the design of the study, collection, analysis and interpretation of data, nor in writing the manuscript.

\section{Availability of data and materials}

All data generated or analysed during this study are included in this published article and its supplementary information files. The transcriptome sequencing raw data has been deposited in NCBI under accession number SRP155137.

\section{Authors' contributions}

YZ carried out the experiment, analyzed the data and drafted the manuscript. KW participated in research design and data analysis. WW, TY, and WD participated in gene cloning and sequence analysis. CX initiated the project, designed the research framework, coordinated the study and participated in writing the manuscript. All authors read and approved the final manuscript.

Ethics approval and consent to participate

Not applicable.

\section{Consent for publication}

Not applicable.

\section{Competing interests}

The authors declare that they have no competing interests.

\section{Publisher's Note}

Springer Nature remains neutral with regard to jurisdictional claims in published maps and institutional affiliations.

Received: 30 May 2018 Accepted: 15 February 2019

Published online: 27 February 2019

References

1. Brookes AJ. The essence of SNPs. Gene. 1999;234(2):177-86.

2. Trick M, Long Y, Meng J, Bancroft I. Single nucleotide polymorphism (SNP) discovery in the polyploidy Brassica napus using Solexa transcriptome sequencing. Plant Biotechnol J. 2009:7(4):334-46.

3. Jehan T, Lakhanpaul S. Single nucleotide polymorphism (SNP) methods and applications in plant genetics: a review. Indian J Biotechnol. 2006;5:435-59.

4. Hiremath PJ, Kumar A, Penmetsa RV, Farmer A, Schlueter JA, Chamarthi SK, Whaley AM, Carrasquilla-Garcia N, Gaur PM, Upadhyaya HD, et al. Largescale development of cost-effective SNP marker assays for diversity assessment and genetic mapping in chickpea and comparative mapping in legumes. Plant Biotechnol J. 2012;10(6):1-17.

5. Garrido-Cardenas JA, Mesa-Valle C, Manzano-Agugliaro F. Trends in plant research using molecular markers. Planta. 2018;247(3):543-57.

6. Mitchell-Olds T, Schmitt J. Genetic mechanisms and evolutionary significance of natural variation in Arabidopsis. Nature. 2006;441(7096): 947-52.

7. Seeb JE, Carvalho G, Hauser L, Naish K, Roberts S, Seeb LW. Singlenucleotide polymorphism (SNP) discovery and applications of SNP genotyping in nonmodel organisms. Mol Ecol Resour. 2011;11(S1):1-8.

8. DePristo MA, Banks E, Poplin R, Garimella KV, Maguire JR, Hartl C, Philippakis AA, Del Angel G, Rivas MA, Hanna M, et al. A framework for variation discovery and genotyping using next-generation DNA sequencing data. Nat Genet. 2011:43(5):491-8.

9. Kumar S, Banks TW, Cloutier S. SNP discovery through next-generation sequencing and its applications. Int J Plant Genomics. 2012;2012:1-15.

10. Davey JW, Hohenlohe PA, Etter PD, Boone JQ, Catchen JM, Blaxter ML. Genome-wide genetic marker discovery and genotyping using nextgeneration sequencing. Nat Rev Genet. 2011;12:499-510. 
11. Yu Y, Wei J, Zhang X, Liu J, Liu C, Li F, Xiang J. SNP discovery in the transcriptome of white Pacific shrimp Litopenaeus vannamei by next generation sequencing. PLoS One. 2014;9(1):e87218.

12. Quinn EM, Cormican P, Kenny EM, Hill M, Anney R, Gill M, Corvin AP, Morris DW. Development of strategies for SNP detection in RNA-Seq data: application to lymphoblastoid cell lines and evaluation using 1000 genomes data. PLoS One. 2013;8(3):e58815.

13. Harismendy O, Ng PC, Strausberg RL, Wang X, Stockwell TB, Beeson KY, Schork NJ, Murray SS, Topol EJ, Levy S, et al. Evaluation of next generation sequencing platforms for population targeted sequencing studies. Genome Biol. 2009;10(3):R32.

14. Rodriguez-Ezpeleta N, Hackenberg M, Aransay MA. Bioinformatics for high throughput sequencing. New York: Springer; 2012.

15. Surget-Groba Y, Montoya-Burgos J. Optimization of de novo transcriptome assembly from next-generation sequencing data. Genome Res. 2010;20(10):1432-40.

16. Liu S, Zhang Y, Zhou Z, Waldbieser G, Sun F, Lu J, Zhang J, Jiang Y, Zhang $H$, Wang $X$, et al. Efficient assembly and annotation of the transcriptome of catfish by RNA-Seq analysis of a doubled haploid homozygote. BMC Genomics. 2013;14:1.

17. McKenna A, Hanna M, Banks E, Sivachenko A, Cibulskis K, Kernytsky A, Garimella K, Altshuler D, Gabriel S, Daly M, DePristo MA. The genome analysis toolkit: a MapReduce framework for analyzing next-generation DNA sequencing data. Genome Res. 2010;20(9):1297-303.

18. Melo ATO, Bartaula R, Hale L. GBS-SNP-CROP: a reference-optional pipeline for SNP discovery and plant germplasm characterization using variable length, paired-end genotyping-by-sequencing data. BMC Bioinformatics. 2016;17(1):29

19. Li H, Handsaker B, Wysoker A, Fennell T, Ruan J, Homer N, Marth G, Abecasis G, Durbin R, 1000 Genome Project Data Processing Subgroup. The sequence alignment/map format and SAMtools. Bioinformatics. 2009;25(16):2078-9.

20. Garrison E, Marth GO. Haplotype-based variant detection from short-read sequencing. 2012. https://arxiv.org/abs/1207.3907v2. Accessed 6 Feb 2019.

21. Li R, Li Y, Fang X, Yang H, Wang J, Kristiansen $K$, Wang J. SNP detection for massively parallel whole-genome resequencing. Genome Res. 2009:19:1124-32

22. Milano I, Babbucci M, Panitz F, Ogden R, Nielsen RO, Taylor MI, Helyar SJ, Carvalho GR, Espineira M, Atanassova M, et al. Novel tools for conservation genomics: comparing two high-throughput approaches for SNP discovery in the transcriptome of the European hake. PLoS One. 2011;6(11):e28008.

23. Zhu PY, He L, Li Y, Huang W, Xi F, Lin L, Zhi Q, Zhang W, Tang YT, Geng C, et al. OTG-snpcaller: an optimized pipeline based on TMAP and GATK for SNP calling from ion torrent data. PLoS One. 2014:9(5):e97507.

24. $\mathrm{Ni} \mathrm{G}$, Strom TM, Pausch $H$, Reimer $C$, Preisinger $R$, Simianer $H$, Erbe $M$. Comparison among three variant callers and assessment of the accuracy of imputation from SNP array data to whole-genome sequence level in chicken. BMC Genomics. 2015;16(1):824.

25. Flicek $P$, Birney $E$. Sense from sequence reads: methods for alignment and assembly. Nat Methods. 2009;6(11):S6-S12.

26. Nielsen R, Paul JS, Albrechtsen A, Song YS. Genotype and SNP calling from next-generation sequencing data. Nat Rev Genet. 2011;12:443-51.

27. You N, Murillo G, Su X, Zeng X, Xu J, Ning K, Zhang S, Zhu J, Cui X. SNP calling using genotype model selection on high-throughput sequencing data. Bioinformatics. 2012;28(5):643-50.

28. Chhangawala S, Rudy G, Mason EC, Rosenfeld AJ. The impact of read length on quantification of differentially expressed genes and splice junction detection. Genome Biol. 2015:16:131.

29. Chang Z, Wang ZJ, Li GJ. The impacts of read length and transcriptome complexity for de novo assembly: a simulation study. PLoS One. 2014;9(4):e94825.

30. Zagordi O, Daumer M, Beisel C, Beerenwinkel N. Read length versus depth of coverage for viral quasispecies reconstruction. PLoS One. 2012;7(10): e47046

31. Chaisson JM, Brinza D, Pevzner AP. De novo fragment assembly with short mate-paired reads: does the read length matter? Genome Res. 2009:19:336-46

32. Jung H, Yoon B, Kim W, Kim D, Hurwood D, Lyons R, Salin K, Kim H, Baek I, Chand $\mathrm{V}$, et al. Optimizing hybrid de novo transcriptome assembly and extending genomic resources for giant freshwater prawns (Macrobrachium rosenbergii): the identification of genes and markers associated with reproduction. Int J Mol Sci. 2016;17(5):690.
33. Nielsen R, Korneliussen T, Albrechtsen A, Li Y, Wang J. SNP calling, genotype calling, and sample allele frequency estimation from new-generation sequencing data. PLoS One. 2012;7(7):e37558.

34. Zhao Y, Dong WQ, Wang K, Zhang B, Allan AC, Lin-Wang K, Chen KS, Xu CJ. Differential sensitivity of fruit pigmentation to ultraviolet light between two peach cultivars. Front Plant Sci. 2017:8:1552.

35. Luo T, Xu K, Luo Y, Chen J, Sheng L, Wang J, Han J, Zeng Y, Xu J, Chen J, et al. Distinct carotenoid and flavonoid accumulation in a spontaneous mutant of ponkan (Citrus reticulata Blanco) results in yellowish fruit and enhanced postharvest resistance. J Agr Food Chem. 2015;63(38):8601-14.

36. Wu GA, Terol J, Ibanez V, López-García A, Pérez-román E, borredá C, Domingo C, Tadeo FR, carbonell-caballero J, Alonso R, et al. Genomics of the origin and evolution of Citrus. Nature. 2018;554:311-6.

37. Maughan PJ, Yourstone SM, Byers RL, Smith SM, Udall JA. Singlenucleotide polymorphism genotyping in mapping populations via genomic reduction and next-generation sequencing: proof of concept Plant Genome. 2010;3:166-78.

38. Wang ZH, Zhuang EJ. Flora of China fruits - peach. Beijing: China Forestry Press; 2001.

39. Chen KS, Li F, Xu CJ, Zhang SL, Fu CX. An efficient macro-method of genomic DNA isolation from Actinidia chinensis leaves. Hereditas. 2004; 26(4):529-31.

40. Shan LL, Li X, Wang P, Cai C, Zhang B, Sun CD, Zhang WS, Xu CJ, Ferguson I, Chen KS. Characterization of CDNAs associated with lignification and their expression profiles in loquat fruit with different lignin accumulation. Planta. 2008:227:1243-54

41. Sambrook J, Fritsch EF, Maniatis T. Molecular cloning: a laboratory manual. 3rd ed. New York: Cold Spring Harbor Laboratory Press; 2001.
Ready to submit your research? Choose BMC and benefit from:

- fast, convenient online submission

- thorough peer review by experienced researchers in your field

- rapid publication on acceptance

- support for research data, including large and complex data types

- gold Open Access which fosters wider collaboration and increased citations

- maximum visibility for your research: over $100 \mathrm{M}$ website views per year

At $\mathrm{BMC}$, research is always in progress.

Learn more biomedcentral.com/submissions 